\title{
PARTICLE ACCELERATION IN EXTRAGALACTIC JETS AND IMPLICATIONS FOR THE HIGH-ENERGY EMISSION FROM
}

\author{
BLAZARS \\ R. SCHLICKEISER \\ Max-Planck-Institut für Radioastronomie, Auf dem Hügel 69, 53121 Bonn, Germany \\ and \\ C. D. DERMER \\ E. O. Hulburt Center for Space Physics, Naval Research Laboratory, Code 7653, \\ Washington, DC 20375-5352, USA
}

September 15, 1993

\begin{abstract}
We demonstrate that the prevalence of superluminal sources in the sample of $\gamma$-ray blazars and the peak of their luminosity spectra at $\gamma$-ray energies can be readily explained if the $\gamma$-rays result from the inverse Compton scattering of the accretion disk radiation by relativistic electrons in outflowing plasam jets. Compton scattering of external radiation by nonthermal particles in blazar jets is dominated by accretion disk photons rather than scattered radiation to distances of $\sim 0.01-0.1 \mathrm{pc}$ from the central engine for standard parameters. The size of the $\gamma$-ray photosphere and the spectral evolution of the relativistic electron spectra constrain the location of the acceleration and emission sites in these objects.
\end{abstract}

Key words: $\boldsymbol{\gamma}$-rays - blazars - emission process - particle acceleration

\section{Introduction}

Since its launch in April 1991, the EGRET experiment on board the Compton Gamma Ray Observatory has increased the number of known $(>5 \sigma)$ extragalactic sources of $>100 \mathrm{MeV} \gamma$-radiation from formerly one (3C273) to 27 (status August 1993, Thompson 1993). With the exception of the LMC, all these sources are radioloud active galactic nuclei (AGNs), of which 5 are classified as BL Lacs and 17 as quasars. Eight of them $(0234+285,0836+710$, Mrk 421, 1156+295, 3C273, 3C279, $1633+382,3 \mathrm{C} 454.3)$ have exhibited apparent superluminal $\left(V_{a p p}>c\right)$ motion components; in a few other cases $(0235+164,0528+134)$, superluminal motion is very likely. The $\gamma$-ray emission in general is variable from time scales as short as 2 days to several months or years. Mrk 421 is the only source that has also been detected at $\mathrm{TeV}$ energies (Punch et al. 1992). Truly remarkable are both the high fraction (>30\%) of known superluminal sources in this sample and the dramatic peak of the luminosity spectra $\nu F_{\nu}$ at $\gamma$-ray frequencies.

\section{A model for the high-energy blazar emission}

Recently, we (Dermer et al. 1992, Dermer and Schlickeiser 1993) have developed a model for the blazar $\gamma$-ray emission based on the relativistic beaming hypothesis (Blandford and Rees 1979) and the unified scenario for AGNs. Simplifying many details, its basic features read as follows: (i) AGNs are powered by accretion of matter onto a black hole; (ii) in the case of radio-loud AGNs, collimated relativistic jets composed of shocks or plasma blobs move with speed $B c$ and bulk Lorentz

T. J.-L. Courvoisier and A. Blecha: Multi-Wavelength Continuum Emission of AGN, 29-32.

(c) 1994 IAU. Printed in the Netherlands. 

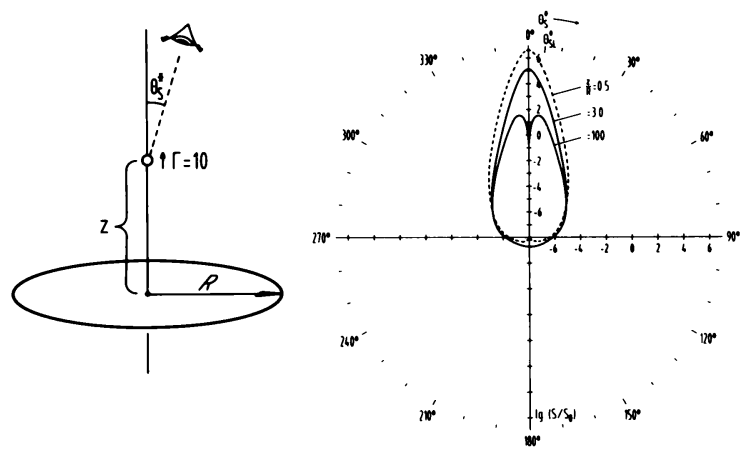

Fig. 1. Diagram illustrating jet model for $\gamma$-ray emission from AGNs (left figure) and radiation beaming pattern (right figure), showing the ratio of scattered luminosity to soft photon luminosity as a function of observing angle $\theta_{s}^{*}$. Different ratios of blob height $z$ to radius $R$ of the soft-photon source are given for a relativistically outflowing plasma blob with bulk Lorentz factor $\Gamma=10$.

factor $\Gamma=\left(1-B^{2}\right)^{-1 / 2}$ outward along the axes of the accretion disk (see left diagram in Fig.1); and (iii) the line-of-sight of the observer determines the AGN classification.

We assume that

(a) a spherical blob of radius $r_{b}$ contains a homogeneous magnetic field of strength $H_{o}$ and relativistic electrons and positrons with an isotropic angular and power-law energy distribution, given by

$$
N(\gamma)=N_{e} \gamma^{-3} \text { for } 1 \leq \gamma_{1} \leq \gamma \leq \gamma_{2}
$$

in the rest frame of the blob;

(b) the relativistic particles are confined to the blob by scattering off intrinsic MHD turbulence;

(c) the blob is of low compactness, and the inverse Compton optical depth $\tau=$ $\sigma_{T} N_{e} r_{b}<1$ is smaller than unity.

\section{3. $\gamma$-ray production}

Among the many possible physical processes in such a system (synchrotron selfCompton, inverse Compton scattering of ambient $2.7 \mathrm{~K}$ microwave background and optical photons, etc.), we have demonstrated (Dermer and Schlickeiser 1993) that the $100 \mathrm{MeV}-10 \mathrm{GeV} \gamma$-rays result from Compton scattering of the ultraviolet and soft $X$-ray accretion disk photons traversing the outflowing blob by the relativistic electrons and positrons in the blob. An important feature of this process is the strong beaming of the scattered $\gamma$-ray emission. Due to Doppler boosting and the strong anisotropy of the accretion disk photons in the comoving fluid frame, the angular distribution of the inverse Compton scattered radiation is very anisotropic and peaks near the superluminal direction $\mu_{S L}^{*}=\cos \theta_{S L}^{*}=B$ once the blob is at 
distances from the central object $z(\mathrm{pc})>\Gamma R=0.003(\Gamma / 10)\left(R / 10^{15} \mathrm{~cm}\right)$, where $R$ denotes the effective size of the accretion disk. From the diagram on the right of Fig. 1, we see that the beaming pattern peaks in the forward direction at values of $z / R<<\Gamma$.

On the basis of estimates for the total electron loss rates in the various interaction processes (see Dermer and Schlickeiser 1993 for details), we find that for parameters appropriate to $3 \mathrm{C} 279$, the inverse Compton scattering of accretion disk photons dominates the production of $\gamma$-rays by

- microwave scattering if $z(\mathrm{pc})<360 \frac{\left(L_{U V} / 10^{46} \mathrm{erg} / \mathrm{s}\right)^{1 / 2}}{(\Gamma / 10)^{2}\left(1+z_{\text {redehift }}\right)^{2}}$,

- Compton scattering of stellar optical photons if $z(\mathrm{pc})<4.3 \frac{\left(L_{U V} / L_{\text {opt }}\right)^{1 / 2}}{(\Gamma / 10)}$,

- the synchrotron-self Compton process if $z(\mathrm{pc})<0.1 \frac{\left(L_{U V} / 10^{46} \mathrm{erg} / \mathrm{s}\right)^{1 / 2}}{\Gamma\left(H_{0} / 1 \mathrm{G}\right)}$,

- Compton scattering of reprocessed radiation in emission line clouds (Sikora et al. 1994) if $z(\mathrm{pc})<0.06 \frac{\left(M_{8}\right)^{1 / 3} R_{E \mu C}^{2 / 3}(1 \mathrm{pc})}{\tau_{-2}^{1 / 3}}$, where $M_{8}$ denotes the central black hole mass in units of $10^{8}$ solar masses, $R_{E L C}$ the distance in units of pc of the emission line clouds from the central black hole, and $\tau_{-2}$ the electron scattering optical depth in emission line clouds in units of $10^{-2}$. Note that the lack of an observed soft X-ray turnover due to photoelectric absorption in contemporaneous GINGA observations of 3C279 (Makino et al. 1993) constrains the value $\tau_{-2} \leq 0.25$. Moreover, we established (see Dermer and Schlickeiser 1994) that for parameters characteristic of $3 \mathrm{C} 279, \gamma-\gamma$ pair annihilation of the produced $1 \mathrm{GeV} \gamma$-radiation is negligible

- with the accretion disk radiation if $z(\mathrm{pc})>4 \cdot 10^{-4} \frac{\left(L_{U V} / 10^{46} \mathrm{erg} / \mathrm{s}\right)^{1 / 2}\left(M_{8}\right)^{1 / 2}}{\left(k_{B} T_{A} / 50 \mathrm{eV}\right)^{1 / 2}}$,

- with reprocessed radiation from emission line clouds at all $z$, since its pair annihilation optical depth is

$$
\tau(1 \mathrm{GeV}) \cong 0.06 E_{\mathrm{GeV}}^{0.7}\left(L_{U V} / 10^{46} \mathrm{erg} / \mathrm{s}\right) \tau_{-2} \ln \left(z / z_{i}\right)<<1
$$

Here $T_{A}$ denotes the temperature of the accretion disk and $z_{i}$ is the distance of the base of the jet from the central source. Estimate (2) is a factor 200 smaller than the estimate of Blandford (1993) because, according to contemporaneous GINGA observations, $3 \mathrm{C} 279$ was in a low-state $(1-10 \mathrm{keV})$ during the $\gamma$-ray flare.

The above constraints place the location of the $\gamma$-ray emission site and the particle acceleration site at distances $10^{15} \mathrm{~cm} \leq z \leq 10^{17} \mathrm{~cm}$ from the central object, where the lower limit is comparable to the radial extent of the accretion disk and is determined by the obvious absence of $\gamma-\gamma$ pair annihilation in the observed $\gamma$-ray spectra, while the upper limit is set by the dominance of other interaction processes which could, however, also contribute to the more slowlyvarying gamma ray emission. Our proposed model of $\gamma$-ray production by inverse Compton scattering of accretion disk photons by outflowing relativistic electrons and positrons in a relativistic jet is very economical: for favorable viewing conditions of the observer with $\theta_{o b s}^{*} \simeq \theta_{S L}^{*}$, we estimate the total energy in relativistic electrons and positrons in the blob to be

$$
E_{t o t} \geq 5 \cdot 10^{46}\left(r_{b} / 10^{15} \mathrm{~cm}\right)^{2}\left(\gamma_{1} / 10\right) \text { erg }
$$




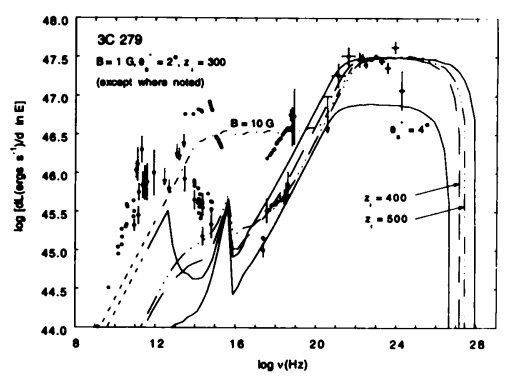

Fig. 2. Model results for the time-integrated luminosity spectrum compared to the multiwavelength observations of $3 \mathrm{C} 279$. The radio-through-optical synchrotron emission, which is probably made farther out in the radio jet, is not modelled here.

Combined with the observed $\gamma$-ray luminosity $L(>100 \mathrm{MeV})=10^{48} L_{48} f \mathrm{erg} \mathrm{s}^{-1}$, where $f$ is the beaming factor, Eq.(3) implies that the intrinsic relativistic electron and positron loss time

$$
t_{R}=E_{t o t} / L(>100 \mathrm{MeV}) \geq 50 L_{48}^{-1}\left(f / 10^{-3}\right)^{-1} \sec
$$

Coupled with the Doppler time-dilation effect, we see from Eq.(4) that very short $\gamma$-ray flare durations can easily be explained in this model if the acceleration time scale is sufficiently short.

The short radiation loss time (4) implies that the time evolution of the electron energy spectrum during the $\gamma$-ray flare is very important. We calculated the timeintegrated $\gamma$-ray emission from a modified Kardashev-model by instantaneously injecting the power law electron distribution (1) at height $z_{i}$, calculating its modification with height in the relativistically outflowing blob due to the operation of the various inverse Compton energy loss processes, and finally integrating the hard $\mathrm{X}$-ray and $\gamma$-ray emissivities over height (Dermer and Schlickeiser 1993). Comparison with multiwavelength observations of $3 \mathrm{C} 279$ is shown in Fig.2. One notices the good fit to the high-energy emission from this source, including the break at $\mathrm{MeV}$ energies which results from incomplete relativistic electron cooling. Moreover the peak of the luminosity spectrum at $\gamma$-ray energies is well reproduced by the model.

\section{References}

Blandford, R.D., Rees, M.J.: 1979, Pittsburg Conference on BL Lac Objects, ed. A. M. Wolfe, Pittsburg, Pittsburg Univ. Press, 328 ,

Blandford, R.D.: 1993, Proceedings of the Compton Symp., ed. N. Gehrels \& M. Friedlander, in press

Dermer, C.D., Schlickeiser, R., Mastichiadis, A.: 1992, AA 256, L27

Dermer, C.D., Schlickeiser, R.: 1993, $A p J$ 416, in press

Dermer, C.D., Schlickeiser, R.: 1994, $A p J S$ 89, in press

Makino, F. et al.: 1993, International Symposium on Neutrino Astrophysics, Takayam/Kamioka, Japan, October, 19-22, 1992 , in press

Punch, M., et al.: 1992, Nat 358, 477

Sikora, M., Begelman, M.C., Rees, M.: 1994, $A p J$, in press

Thompson, D.J.: 1993, this conference, 